\title{
Magnetocaloric effect as a signature of quantum level-crossing for a spin-gapped system
}

\author{
Tanmoy Chakraborty ${ }^{1,2}$, Chiranjib Mitra ${ }^{1}$ \\ ${ }^{1}$ Indian Institute of Science Education and Research (IISER) Kolkata, Nadia-741246, West Bengal, India and \\ ${ }^{2}$ Institute for Materials Research (IMO), Hasselt University, \\ Wetenschapspark 1, B-3590 Diepenbeek, Belgium
}

\begin{abstract}
Recent research dealing with magnetocaloric effect (MCE) study of antiferromagnetic (AFM) low dimensional spin systems have revealed a number of fascinating ground-state crossover characteristics upon application of external magnetic field. Herein, through MCE investigation we have explored field-induced quantum level-crossing characteristics of one such spin system: $\mathrm{NH}_{4} \mathrm{CuPO}_{4} \cdot \mathrm{H}_{2} \mathrm{O}(\mathrm{NCP})$, an AFM spin 1/2 dimer. Experimental magnetization and specific heat data are presented and the data have been employed to evaluate entropy, magnetic energy and magnetocaloric properties. We witness a sign change in magnetic Grüneisen parameter across the level-crossing field $B_{C}$. An adiabatic cooling is observed at low temperature by tracing the isentropic curves in temperature-magnetic field plane. Energy-level crossover characteristics in NCP interpreted through MCE analysis are well consistent with the observations made from magnetization and specific heat data.
\end{abstract}

Studying low dimensional quantum antiferromagnets (AFM) has been at the forefront of both experimental and theoretical condensed matter physics due to novel nature of their ground states. This has led to a number of fascinating physical properties [1 8]. Spin-gapped compounds are a class of quantum spin systems, which possess an energy gap in the excitation spectra [9]. Depending on the nature of the inter-dimer coupling AFM spin dimers exhibit various kinds of quantum phenomena like Bose-Einstein condensation of magnons [10, 11, appearance of magnetization plateaus [12, 13] etc. under the influence of magnetic field, an external tuning parameter. Spin dimer systems with significantly weak interdimer interaction can be considered as independent spin clusters. The energy spectrum of an AFM spin $1 / 2$ dimer consists of a singlet ground state and a 3 -fold degenerate state which upon application of external magnetic field splits into three states and evolve as the field changes. A level-crossing between the ground state and the first excited state occurs when the field is increased through a critical field value. Thus, the ground state undergoes a qualitative change and the first excited state becomes the new ground state. Such level-crossing happens between two pure quantum states which ideally is a zero temperature phenomena, although it is possible to capture its evidence by measuring the physical properties of the system at finite temperature.

Here we have demonstrated magnetocaloric effect (MCE) at field-induced energy level-crossing in $\mathrm{NH}_{4} \mathrm{CuPO}_{4} \cdot \mathrm{H}_{2} \mathrm{O}$ (NCP), a Heisenberg dimer system. MCE is an intrinsic property of magnetic systems which in general refers to a change in sample temperature $\left(T_{\text {Samp }}\right)_{S}$ upon adiabatic change of applied magnetic field 14. Thus MCE is associated with an isothermal change in entropy with field which is expressed as $(\partial T / \partial H)_{S}=-\left[T(\partial S / \partial B)_{T}\right] / C$, where $C$ is the specific heat in constant field. MCE has been widely exploited in refrigeration applications both in cryogenic [15] and room temperature [16]. Having larger change in entropy
$(\Delta S / \Delta B)_{T}$ and $\left(T_{S a m p}\right)_{S}$ in isothermal and adiabatic process respectively are the characteristics of superior magnetocaloric materials which have the potential for novel cooling applications. In this context, paramagnetic salts can act as suitable substitutes for ${ }^{3} \mathrm{He}-{ }^{4} \mathrm{He}$ dilution refrigerant in cooling technology. To avail temperature in $\mathrm{mK}$ range [17, magnetic refrigeration has potential applications in space technology [18, medical sciences 19 and so on.

MCE has been efficiently employed to explore the low temperature behavior of a variety of systems like rareearth transition-metal-based magnetic compounds [20, manganite 21], Heusler alloy [22], plastic crystals [23], intermetallic compounds [24, low dimensional spins systems like spin chain [7, 25, 26], spin ladders [27] and so on. It has been observed that an enhancement in MCE occurs being triggered by geometric frustration in different spin lattice systems which can facilitate cryogenic cooling applications [28]. For systems undergoing a level crossing under the influence of magnetic field, study of MCE can reveal novel qualitative behavior of various thermodynamic parameters in the vicinity of the quantum critical point. A few experimental studies have focused on exploring quantum critical properties by studying $\mathrm{MCE}$ of spin $1 / 2$ chain compounds [7, 29 31. In the present work, MCE is investigated for NCP by measuring magnetic and thermal properties at finite temperature. Main motivations of the present study is exploring the thermodynamic behavior of NCP in detail and witnessing the field-induced level crossing by capturing magnetocaloric response. We have also discussed the possibility for application of $\mathrm{NCP}$ as an adiabatic demagnetization refrigerant.

The crystallographic analysis of the compound showed layers of centrosymmetric $\mathrm{Cu}_{2} \mathrm{O}_{8}$ dimers which are cross connected with each other by phosphate tetrahadra [32]. Fig. 1 schematically exhibits the arrangement of the $\mathrm{Cu}_{2} \mathrm{O}_{8}$ dimers in a layered structure and the four spin exchange (EP) pathways. Spin dimer analysis through 


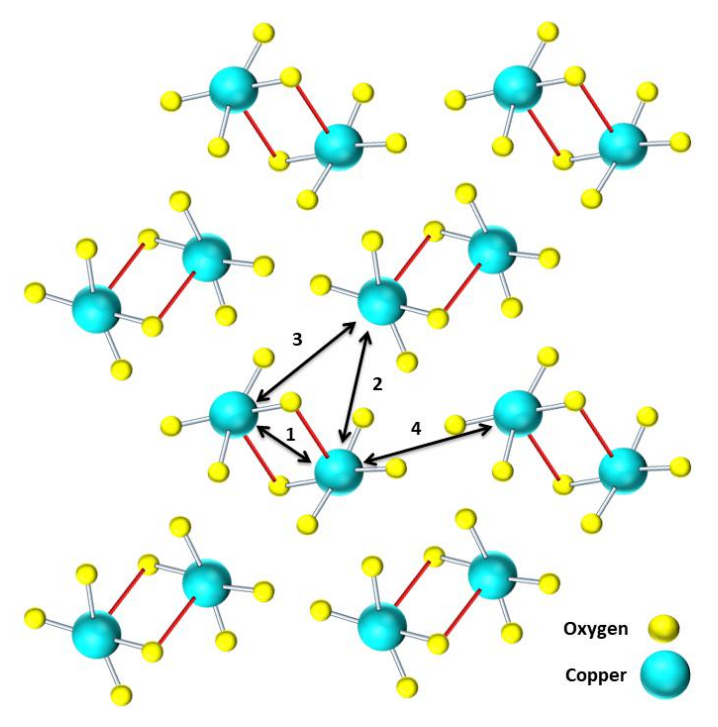

FIG. 1. A schematic diagram of $\mathrm{Cu}_{2} \mathrm{O}_{8}$ dimers distributed in a layer in $\mathrm{NH}_{4} \mathrm{CuPO}_{4} \cdot \mathrm{H}_{2} \mathrm{O}$ crystal. Four spin exchange pathways 1, 2, 3 and 4 are shown by the black arrows, the oxygen and the copper atoms are represented by the yellow and cyan spheres respectively. The details of the crystal structure can be found in ref. 33.

extended Hückel tight binding calculations [33, combined with crystallographic analysis 32 suggest, and it has been experimentally proven [34 that being the intradimer interaction (EP1) significantly larger than the interdimer interactions (EP2, 3 and 4), the system can be best described by isolated spin $1 / 2$ Heisenberg dimer model, where the two spins are antiferromagnetically coupled to each other via a strong intradimer exchange coupling constant $J$. Hence, one can ignore the terms associated with EP2, 3 and 4 in the Hamiltonian. The Hamiltonian for a dimer can be expressed as [35]:

$$
H_{\text {dimer }}=J \overrightarrow{S_{1}} \cdot \overrightarrow{S_{2}}+g_{e} \mu_{B} B\left(S_{1}^{Z}+S_{2}^{Z}\right),
$$

Here $\vec{S}_{i}(i=1,2)$ is the vector spin operator at $i$-th site, $g_{e}$ is the Landé g-factor, $\mu_{B}$ is the Bohr magneton and $B$ is the applied magnetic field. $J$ is expressed in unit of $K$. The energy spectrum of the system consists of a singlet ground state $\frac{1}{\sqrt{2}}(\uparrow \downarrow-\downarrow \uparrow)$ with energy eigenvalue $\frac{-3 J}{4}$ and triplet excited states $\uparrow \uparrow, \downarrow \downarrow$ and $\frac{1}{\sqrt{2}}(\uparrow \downarrow+\downarrow \uparrow)$ with eigenvalues $\frac{J}{4}-g_{e} \mu_{B} B, \frac{J}{4}+g_{e} \mu_{B} B$ and $\frac{J}{4}$ respectively 35.

NCP was synthesized and Greenish-blue single crystals were crystallized following the preparation route described in ref. 34. To perform a detail magneto-thermal analysis of NCP, magnetization and specific heat measurements were carried out in the temperature range, where the short range AFM correlations are persistent, and in the magnetic field range, where one can capture the closing and reopening of the spin excitation gap. Temperature and magnetic field dependent mag- netic measurements were performed in a SQUID (Superconducting Quantum Interference Device) based magnetometer by Quantum Design, USA in the temperature and magnetic field range of $2-10.2 \mathrm{~K}$ and $0-9 \mathrm{~T}$ respectively. In the same temperature and field range we performed the heat capacity measurements of NCP by means of $\mathrm{AC}$ (alternating current) calorimetry technique in a cryogen-free magnetic system manufactured by Cryogenic limited, UK.

Fig. 2 summarizes the results of the thermodynamic measurements and shows some of the measured data along with the theoretical curves for dimer model. Temperature depnedent magnetization $(M)$ data measured at an applied field of $0.1 \mathrm{~T}$ is fitted to the analytical expression for spin $1 / 2$ dimer model described in ref. 34. Fig. 2 (a) shows the $M(T)$ data along with the fitted curve. Fig. 2(b) depicts the magnetization isotherms measured at $2 \mathrm{~K}, 3 \mathrm{~K}, 6 \mathrm{~K}, 8 \mathrm{~K}$ and $10.2 \mathrm{~K}$ and their fit to the expressions for corresponding magnetization isotherms derived for spin 1/2 dimer Hamiltonian shown in Eq. (1) and discussed in ref. 4. The fitting analyses yielded exchange coupling constant $J=5 \mathrm{~K}$. A step-like behavior associated with a jump from one plateau to another could be observed in the magnetization curves especially at lower temperature which corresponds to the levelcrossing of singlet ground state and the excited state $\uparrow \uparrow$. The peak values in the field derivative of magnetization $(\partial M / \partial H)_{T}$ isotherms in Fig. 2(c) indicate that the critical field is $\sim 7 \mathrm{~T}$. The jump in $[M(H, T), H]_{T}$ curves gets less pronounced and the peaks in $(\partial M / \partial H)_{T}$ curves get more broadened at higher temperature as the relative weight of the singlet state decreases in the statistical mixture of all four states at higher temperatures [34, 36. Magnetic contribution to the molar specific heat $\left(C_{m}\right)$ data has been extracted by subtracting the lattice part from the measured specific heat data following the similar way described in ref. 34. We obtained Debye temperature $\theta_{D}=101.9 \mathrm{~K}$. The measured specific heat $C(T)$ can be expressed as an addition of three terms: $C(T)=C_{m}(T)+\gamma T+\beta T^{3}$ where $\gamma$ is Sommerfeld coefficient and $\beta$ is responsible for lattice contribution. We showed earlier that NCP being a magnetic insulator, electronic specific heat does not have any contribution to $C(T)$ 34. When we plotted the experimental data $\frac{C(T)-C_{m}(T)}{T}$ vs. $T^{2}$, it turned out to be a straight line that passes through the origin which proves that $\gamma=0$ for NCP. $C_{m}$ vs. $T$ data for fixed magnetic field values of $0 \mathrm{~T}, 2 \mathrm{~T}, 3 \mathrm{~T}, 4 \mathrm{~T}$ and $5 \mathrm{~T}$ were fitted to the expression for dimer model $C_{m}=1 / k_{B} T^{2}\left(\left\langle H_{\text {dimer }}^{2}\right\rangle-\left\langle H_{\text {dimer }}\right\rangle^{2}\right)$ 37. for the corresponding field values with $J$ as the fitting parameter. We obtained $J=5 \mathrm{~K}$ which is consistent with the earlier analysis. The experimental data along with the fitted curves are shown in Fig. 2(d). The broad peaks in $\left[C_{m}(H, T), T\right]_{H}$ plots signify Schottkylike anomaly which arises due to gradual occupation of the energy states upon increasing temperature [34, 37. An excellent match between the experimental $C_{m}(H, T)$ and $M(H, T)$ data, and their corresponding theoretical 
fits ascertain that NCP is a very good representative of Heisenberg spin $1 / 2$ dimer model.

Next, estimation of magneto-thermal properties like magnetic energy and entropy are performed using the abovepresented data, important magneto-caloric parameters have been calculated and their distinct features have been discussed. $C_{m}(H, T)$ vs $T$ data-sets measured at different fields have been substituted in the equation $S_{m}(T)=\left(S_{m}\right)_{2 K}+\int_{2 K}^{10.2 K}\left[C_{m}\left(H, T^{\prime}\right) / T^{\prime}\right] d T^{\prime}$ [38] to calculate magnetic entropy $S_{m}(T)$ as a function of temperature for different constant fields. $\left(S_{m}\right)_{2 K}$ for corresponding fields have been determined theoretically for dimer model and incorporated in the integration. The entropy of a dimer gradually increases as the system evolves from a populated singlet state at lower $T$ to the configuration where of the all four eigenstates are thermally populated at higher $T$ with the occupancy given by the Boltzmann factor. When $B=J / g_{e} \mu_{B}$, the ground state has a two fold degeneracy which is associated with an amount of $R \ln 2$ change in entropy per dimer where $R$ is the universal gas constant. However, when $B \neq J / g_{e} \mu_{B}$, the entropy reaches a saturation value of $R \ln 4$. This is in good analogy with our experimental data exhibited in Fig. 2(e). By using the equation $U_{m}(T)=\left(U_{m}\right)_{2 K}+\int_{2 K}^{10.2 K} C_{m}\left(H, T^{\prime}\right) d T^{\prime} 38$ numerical integration on $C_{m}(H, T)$ data is carried out to calculate magnetic energy $U_{m}(T)$. Fig. $2(\mathrm{f})$ exhibits $U_{m}(T)$ vs. $T$ for different magnetic field values. The constant $\left(U_{m}\right)_{2 K}$ for different magnetic field values are calculated theoretically. When we consider the evolution of magnetic energy of a dimer as a function of field, the ground state energy $\left(E_{g}=-3 J / 4\right)$ is constant below level crossing $\left(B_{C} \sim 7 T\right)$. However, when $B>B_{C}$, $E_{g}\left(=-g_{e} \mu_{B} B+J / 4\right)$ starts varying with field. This change in $E_{g}$ is sharp at $0 \mathrm{~K}$, but gets more gradual at higher temperature due to thermal population in all four states. Consequently, the constant field $U(T)$ curves in Fig. 2(f) starts from nearly equal values at $2 \mathrm{~K}$ whereas the starting values increases in the lower side as $B$ increases beyond $7 \mathrm{~T}$.

An important quantity to capture the crossing of energy levels is Grüneisen ratio [39, 40, that provides a measure of cooling efficiency of the system upon adiabatic change in magnetic field. In case of magnetic field induced transition, one can define the magnetic Grüneisen ratio $\Gamma_{B}$ as the adiabatic change in temperature upon changing the magnetic field, which equals the ratio of negative temperature derivative of magnetization to heat capacity at constant field through the following Maxwell's thermodynamic relations.

$$
\Gamma_{B}=\left(\frac{d M}{d T}\right)_{B} / C_{B}=\frac{1}{T}\left(\frac{\partial T}{\partial B}\right)_{S}
$$

Hence, in fact, an estimation of $\Gamma_{B}$ gives a quantitative measure of adiabatic MCE. As the system passes through the critical point upon sweeping the magnetic field, $\Gamma_{B}$ changes its sign and exhibits divergence. Thus an investigation of adiabatic MCE can be useful in captur- ing the critical field. Instead of directly measuring the adiabatic change in sample temperature with magnetic field, $\Gamma_{B}$ can alternatively be estimated for a given field change from $C_{m}(H, T)$ and $M(H, T)$ data by employing the above Maxwell's relations [Eq. (2)] [41. Using this method we have estimated MCE for NCP. Experimental quantification of $\Gamma_{B}$ for different temperatures ranging from $2 \mathrm{~K}$ to $10.2 \mathrm{~K}$ were performed and are shown in Fig. 3. As the field increases, the plotted $\Gamma_{B}$ values go through a minima at $B \sim 5 T$ and changes its sign from negative to positive in the vicinity of the levelcrossing point. When $\Gamma_{B}$ changes its sign, an occurrence of maximum thermal entropy happens because of competing ground states $\frac{1}{\sqrt{2}}(\uparrow \downarrow-\downarrow \uparrow)$ and $\uparrow \uparrow$ in the field region $B<B_{C}$ and $B>B_{C}$ respectively, which consequently creates a frustration in the system. Upon increasing the field further, $\Gamma_{B}$ passes through a maximum when $B \sim 8 T$. Below $B_{C}$, the negative values of $\Gamma_{B}$ signifies adiabatic cooling due to magnetization, whereas an enhancement in $\Gamma_{B}$ above $B_{C}$ signifies cooling through demagnetization. The contrast of the $\Gamma_{B}(B)$ curve is higher at lower $T$ because, when $T$ decreases the statistical weight of the singlet ground state $\frac{1}{\sqrt{2}}(\uparrow \downarrow-\downarrow \uparrow)$ increases in the thermal mixture of the all four states. With decreasing $T$ the minima and maxima at $B \sim 5 T$ and $8 T$ gets sharper and eventually would diverge at $T=0$ due to the field induced critical behavior of Heisenberg spin system NCP. This diverging property of $\Gamma_{B}$ for NCP in the both sides of the critical point is consistent with the observation made for $K C u F_{3}$, another spin $1 / 2$ quantum critical system [30]. The sign change in $\Gamma_{B}$ is more drastic at lower temperatures and centered close to $B_{C}$ which enables one to identify the level crossing point. Thus one can note the critical field $B_{C} \sim 7 \mathrm{~T}$ for NCP.

An interactive way to trace the sample temperature upon sweeping the field and thus capturing the critical point is to represent the quantity $(d T / d B)_{s}$ in a contour plot. We have used the experimental thermodynamic data in Eq. (2) to quantify $(d T / d B)_{s}$ and generated a contour plot shown in Fig. 4(a) along with the simulation for spin dimer in Fig. 4(b). The Hamiltonian mentioned in Eq. (1) was used to simulate magnetization and specific heat, which were eventually used to calculate $(d T / d B)_{s}$ using the Eq. (2). We used MATLAB for the simulations. Both the plots are depicted in the same temperature (2 to $10.2 \mathrm{~K}$ ) and field (0 to $9 \mathrm{~T}$ ) range and they exhibit a very good resemblance. Inset of Fig. 4 (b) shows the simulation of $(d T / d B)_{s}$ through $B_{C}$ down to $0 \mathrm{~K}$. A minimum in $(d T / d B)_{s}$ suggests an enhanced MCE close to the $B_{C}$ which are evident both from the experimental and theory plots.

The observation of substantial variation in $\Gamma_{B}$ with field from the last analysis indicates that NCP can exhibit significant change in temperature with small variation of magnetic field which motivates us to examine its performance as magnetic refrigerant. Using the experimental thermodynamic data $C_{m}(H, T)$ and $M(H, T)$ we have quantified the adiabatic variation of sample temperature 
(a)
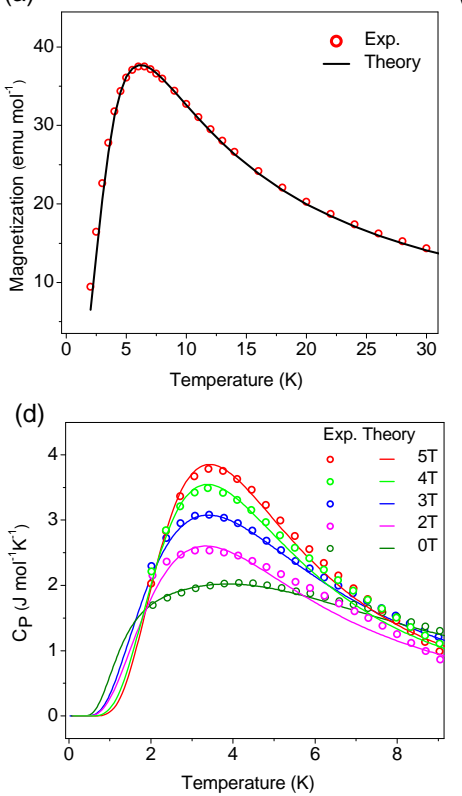

(b)

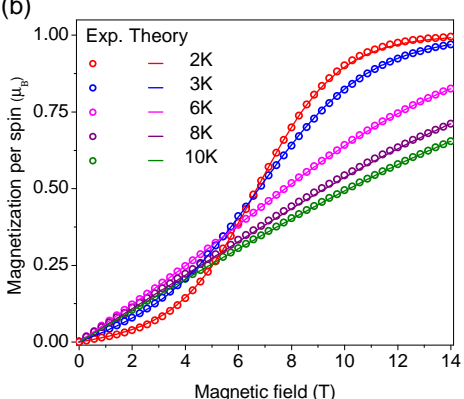

(e)

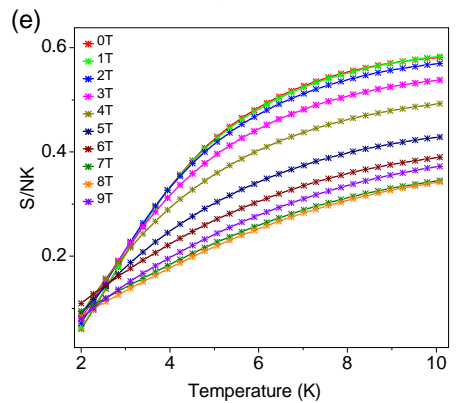

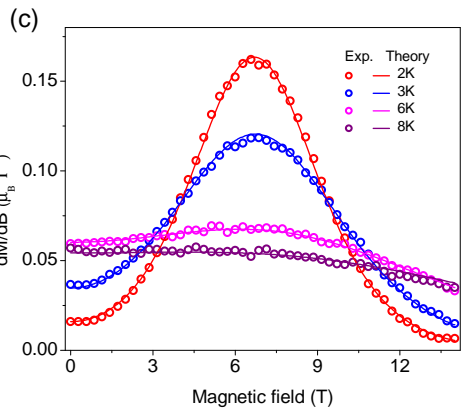

(f)

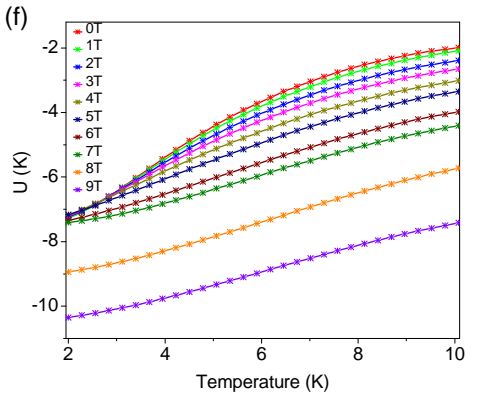

FIG. 2. (a) Temperature dependent magnetization data measured at an applied magnetic field of 0.1T. (b) Field dependent isothermal magnetization data at different temperatures, (c) field derivative of the magnetization isotherms and (d) temperature dependent magnetic specific heat data at different fields along with the theoretical curves for dimer model as decribed in the main text. The symbols indicate the data and the solid lines signify theoretical curves. (e) and (f) show estimated entropy and internal energy respectively from experimental specific heat data at various fields as mentioned in the legend.

in response to change in field by employing the following thermodynamic relation 41]

$$
\triangle T=T_{f}-T_{i}=-\int_{H_{i}}^{H_{f}} \frac{T(H)}{C_{m}(H, T)}\left[\frac{\partial M(H, T)}{\partial T}\right]_{H} d H
$$

Here $H_{i}$ and $H_{f}$ are the initial and final magnetic fields which correspond to initial and final sample temperature $T_{i}$ and $T_{f}$ [42]. Quantified experimental constant-entropy curves for entropy varying from $S / R=0.061$ to 0.435 correspond to different initial temperatures starting from $2 \mathrm{~K}$ to $5.2 \mathrm{~K}$ are plotted in $T-B$ plane and are shown in Fig. 5(a). The plot reveals that the highest variation in $T_{S}$ with field happens for the curve with $S / R=0.061$ which signifies that cooling is maximum when the initial value of $T_{S}$ is minimum. Fig. 5(b) exhibits the evolution of the four eigenstates with field and the crossover between the $\frac{1}{\sqrt{2}}(\uparrow \downarrow-\downarrow \uparrow)$ and $\uparrow \uparrow$ states. The lowest entropy isentrope shows a minimum in the vicinity of the level crossing field signifying a noticeable magnetocaloric response at the closing of the spin gap which captures the signature of quantum criticality in NCP. Energy level crossover properies in NCP interpreted from the MCE is consistent with the observation made from magnetization data discussed above. NCP exhibits adiabatic cooling over a wide range of temperature although the minima in the isentropes get less sharper and the cooling is less along the curves with higher values of $S / R$ as shown in Fig. 5(a). The curve with $S / R=0.435$ shows minimum variation in $T_{S}$ with field. This MCE study shows that we could achieve a cooling down to a temperature as low as $0.7 \mathrm{~K}$ for the minimum initial temperature of $2 \mathrm{~K}$.

Although in this report the MCE investigation for $\mathrm{NCP}$ is not performed at sufficiently low temperatures, a notable adiabatic cooling response for $T_{i}=2 \mathrm{~K}$ indicates that it can be a possible candidate for coolant applications in low temperature. By the virtue of being an AFM critical spin system, it readily has advantages over paramagnetic salts as coolant: a distinct excitation spec- 


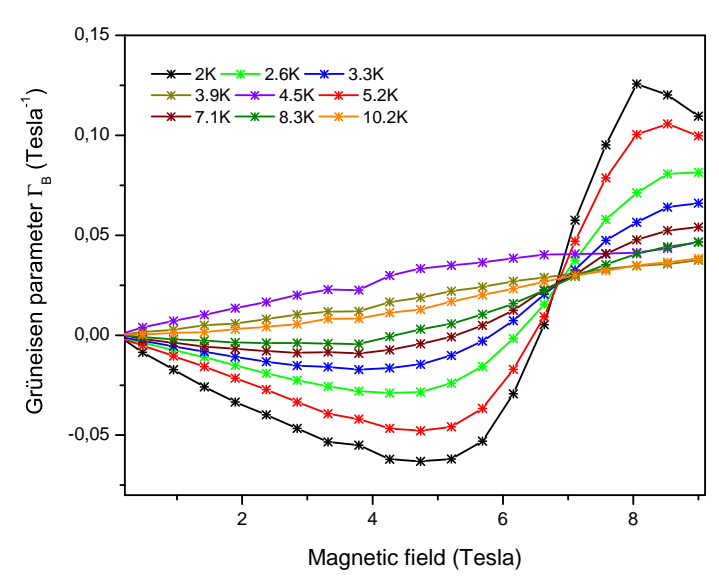

FIG. 3. Experimental magnetic Grüneisen ratio $\Gamma_{B}$ calculated from measured $C_{m}(H, T)$ and $M(H, T)$ data for NCP. $\Gamma_{B}$ values are plotted as a function of applied magnetic field for different constant temperature.

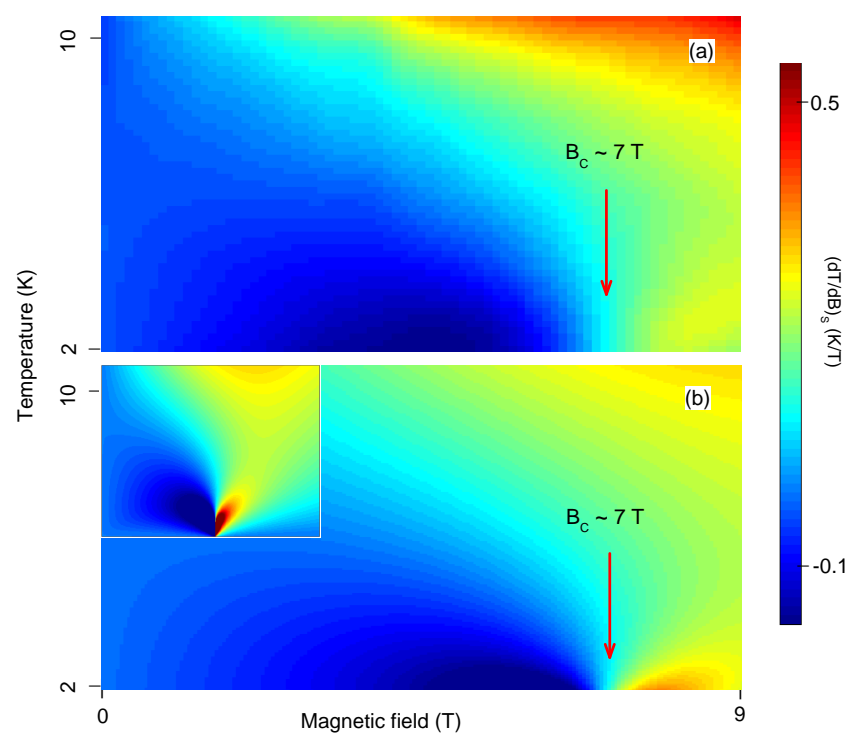

FIG. 4. (a) Experimental and (b) theoretical (dimer) $(d T / d B)_{S}$ represented in contour plots in $T-B$ plane. Both the plots are shown in same range: $T=2 \mathrm{~K}$ to $10.2 \mathrm{~K}$ and $B=0 \mathrm{~T}$ to $9 \mathrm{~T}$. The plot shown in inset exhibits the features of $(d T / d B)_{S}$ plot for dimer model down to $0 \mathrm{~K}$.

trum of such spin systems allows low energy magnetic excitations above the level-crossing point which makes it possible to achieve significant cooling 30. Specific heat properties of NCP enables the system to enhance the ability to absorb heat with warming them up in a slow rate. Furthermore, the system can be prepared following a simple synthesis route and can be crystallized into regular shaped, mechanically rigid, vapor and air-stable single crystals in less than one month. These properties indicate that close to the level-crossing point, NCP can

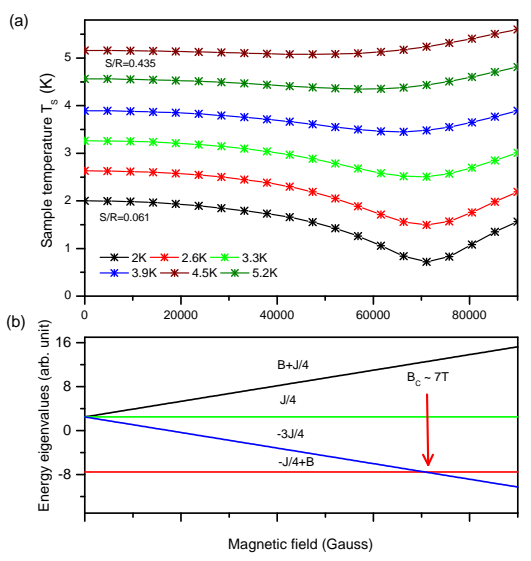

FIG. 5. (a) Experimental isentropes for NCP where $S / R$ varies from 0.061 to 0.435 . The curves represent adiabatic cooling of the system upon sweeping the magnetic field. (b) Simulated energy spectrum for dimer shows the magnetic field induced energy level crossing around a critical field value of 7T. At the same field we observed maximum cooling in the isentrope with lowest initial starting temperature of $2 \mathrm{~K}$.

be a possible material for cooling applications over a wide temperature range. However, still a few more investigations like studying thermal conductivity and estimating the achievable base temperature are necessary to test the efficiency of NCP as a magnetic coolant. We plan to perform these investigations in details in future.

To summarize, through MCE study certain characteristics of magnetic field induced level-crossing in an AFM spin gapped system have been captured. We have carried out a detail study of experimental thermodynamic properties and showed their analogy to the simulation for Heisenberg spin 1/2 dimer model. Experimental magnetization, specific heat, magnetic energy and entropy as a function of temperature and magnetic field have been investigated. The system exhibits a notable MCE in the vicinity of the critical magnetic field where the transition between singlet ground state and triplet first excited state happens. Thus, tracing the experimental isentropes we could determine the critical field for NCP which turned out to be $\sim 7 \mathrm{~T}$. A noticeable amount of MCE is observed over a wide temperature range. The demonstration of adiabatic cooling of NCP opens up a possibility for applying the material as a refrigerant in coolant technology. 


\section{ACKNOWLEDGMENTS}

The authors would like to thank the Ministry of Human Resource Development (MHRD), Government of India, for funding.

[1] T. Giamarchi, Quantum physics in one dimension, vol. 121 (Oxford university press, 2004).

[2] P. Lemmens, G. Güntherodt, and C. Gros, Phys. Rep 375, 1 (2003).

[3] M. Arnesen, S. Bose, and V. Vedral, Phys. Rev. Lett. 87, 017901 (2001).

[4] D. Das, H. Singh, T. Chakraborty, R. K. Gopal, and C. Mitra, New J. Phys. 15, 013047 (2013).

[5] T. Giamarchi, C. Rüegg, and O. Tchernyshyov, Nat. Phys 4, 198 (2008).

[6] F. He, Y. Jiang, Y.-C. Yu, H.-Q. Lin, and X.-W. Guan, Phys. Rev. B 96, 220401 (2017).

[7] O. Breunig, M. Garst, A. Klümper, J. Rohrkamp, M. M. Turnbull, and T. Lorenz, Sci. adv 3, eaao3773 (2017).

[8] A. Rahmani, A. E. Feiguin, and C. D. Batista, Phys. Rev. Lett. 113, 267201 (2014).

[9] I. Bose, Curr. Sci. 88, 62 (2005).

[10] C. Rüegg, N. Cavadini, A. Furrer, H.-U. Güdel, K. Krämer, H. Mutka, A. Wildes, K. Habicht, and P. Vorderwisch, Nature 423, 62 (2003).

[11] S. Sebastian, N. Harrison, C. Batista, L. Balicas, M. Jaime, P. Sharma, N. Kawashima, and I. Fisher, Nature 441, 617 (2006).

[12] H. Kageyama, K. Yoshimura, R. Stern, N. Mushnikov, K. Onizuka, M. Kato, K. Kosuge, C. Slichter, T. Goto, and Y. Ueda, Phys. Rev. Lett. 82, 3168 (1999).

[13] K. Kodama, M. Takigawa, M. Horvatić, C. Berthier, H. Kageyama, Y. Ueda, S. Miyahara, F. Becca, and F. Mila, Science 298, 395 (2002).

[14] A. M. Tishin and Y. I. Spichkin, The magnetocaloric effect and its applications (CRC Press, 2016).

[15] K. A. GschneidnerJr, V. Pecharsky, and A. Tsokol, Rep. Prog. Phys 68, 1479 (2005).

[16] O. Tegus, E. Brück, K. Buschow, and F. De Boer, Nature 415, 150 (2002).

[17] O. Lounasmaa, Academic Press, London pp. 156-159 (1974).

[18] W. Chen, Cryogenics 62, 206 (2014).

[19] J. Li, Y. Qu, J. Ren, W. Yuan, and D. Shi, Nanotechnology 23, 505706 (2012).

[20] M. Das, S. Roy, N. Khan, and P. Mandal, Phys. Rev. B 98, 104420 (2018).

[21] M. Quintero, J. Sacanell, L. Ghivelder, A. Gomes, A. Leyva, and F. Parisi, Appl. Phys. Lett. 97, 121916 (2010).
[22] K. Synoradzki, K. Ciesielski, and D. Kaczorowski, Acta Phys. Pol. A 133, 691 (2018).

[23] B. Li et al., Nature 567, 506 (2019).

[24] A. Sankar, J. A. Chelvane, A. Morozkin, A. Nigam, S. Quezado, S. Malik, and R. Nirmala, AIP Adv. 8, 056208 (2018).

[25] M. Zhitomirsky and A. Honecker, J. Stat. Mech. Theory Exp 2004, P07012 (2004).

[26] A. Honecker and S. Wessel, Condensed Matter Physics 12, 399 (2009).

[27] F. Amiri, S. Mahdavifar, H. Hadipour, and M. S. Naseri, J. Low Temp. Phys. 177, 203 (2014).

[28] M. Zhitomirsky, Phys. Rev. B 67, 104421 (2003).

[29] J.-S. Xiang, C. Chen, W. Li, X.-L. Sheng, N. Su, Z.H. Cheng, Q. Chen, and Z.-Y. Chen, Sci Rep. 7, 44643 (2017).

[30] B. Wolf, Y. Tsui, D. Jaiswal-Nagar, U. Tutsch, A. Honecker, K. Remović-Langer, G. Hofmann, A. Prokofiev, W. Assmus, G. Donath, et al., Proc. Natl. Acad. Sci. 108, 6862 (2011).

[31] J. W. Sharples, D. Collison, E. J. McInnes, J. Schnack, E. Palacios, and M. Evangelisti, Nat. Commun. 5, 5321 (2014).

[32] A. Pujana, J. L. Pizarro, L. Lezama, A. Goñi, M. I. Arriortua, and T. Rojo, J. Mater. Chem 8, 1055 (1998).

[33] H. Koo and M. Whangbo, J. Solid State Chem 181, 276 (2008)

[34] T. Chakraborty, H. Singh, and C. Mitra, J. Appl. Phys. 115, 034909 (2014).

[35] J. B. Parkinson and D. J. Farnell, An introduction to quantum spin systems, vol. 816 (Springer, 2010).

[36] T. Chakraborty, H. Singh, and C. Mitra, J. Magn. Magn. Mater 396, 247 (2015).

[37] H. Singh, T. Chakraborty, D. Das, H. Jeevan, Y. Tokiwa, P. Gegenwart, and C. Mitra, New J. Phys. 15, 113001 (2013).

[38] T. Chakraborty, Ph.D. thesis, Indian Institute of Science Education and Research Kolkata (2015).

[39] N. Vočadlo and G. D. Price, Phys. Earth Planet. Inter. 82, 261 (1994).

[40] P. G, Rep. Prog. Phys 79, 114502 (2016).

[41] V. Pecharsky, K. Gschneidner Jr, A. Pecharsky, and A. Tishin, Physical review B 64, 144406 (2001).

[42] A. Tishin, K. Gschneidner Jr, and V. Pecharsky, Phys. Rev. B 59, 503 (1999). 